\title{
La Reflexión Colectiva entre Profesoras en Formación Inicial y Continua como Espacio de Construcción de una Pedagogía Inclusiva
}

\section{The Collective Reflection among Teachers in Initial and Ongoing Teacher Education as a Space for Building an Inclusive Pedagogy}

\author{
Constanza Herrera-Seda * \\ Carlos Vanegas-Ortega \\ Esteban Vicencio-Callejas \\ Katalina Maldonado-Amaro
}

Universidad de Santiago de Chile, Chile

\begin{abstract}
En las últimas décadas construir una pedagogía inclusiva se ha convertido en un objetivo fundamental para el sistema educativo chileno y la formación de profesores en una tarea insoslayable que implica importantes desafíos. El objetivo de este estudio fue describir el aporte de la reflexión colectiva sobre la práctica entre profesores en formación inicial y continua para la construcción de una pedagogía inclusiva, a partir de los principios del diseño universal para el aprendizaje. Para ello, se elaboró un modelo conceptual de formación docente para la inclusión y se abordó metodológicamente desde un enfoque cualitativo de investigación-formación-acción. Este trabajo muestra el diseño e implementación de un plan de formación docente aplicado a 5 equipos de trabajo conformados por 2 profesoras en formación inicial y una profesora en formación continua. Con base en los datos recopilados mediante entrevistas, cuestionarios y grabaciones de sesiones de trabajo conjunto se establecieron resultados sobre las convergencias y divergencias vivenciadas por las participantes. Las principales conclusiones corroboran la relevancia de la reflexión colectiva entre las profesoras para el desarrollo profesional en estas dos etapas y para promover la transformación de las prácticas de aula hacia la inclusión educativa.
\end{abstract}

Descriptores: Educación inclusiva; Formación de profesores; Práctica pedagógica; Trabajo en equipo; Reflexión colaborativa.

In the last few decades to build an inclusive pedagogy has become a fundamental goal for the Chilean educational system, and teacher education into an unavoidable task which involves relevant challenges. The objective of this research was to describe the contribution of the collective reflection on practice among teachers in initial and ongoing training to build an inclusive pedagogy, departing from the universal design for learning principles. With this aim, a conceptual framework of teacher education for inclusion was elaborated, and it was methodologically addressed with a qualitative approach of training-action-research. This work shows the design and implementation of a teacher training program applied to 5 work teams formed by 2 pre-service teachers and one in-service teacher. Based on the data collected through interviews, questionnaires, and work session records the results about the convergences and divergences experienced by participants was established. The main conclusions corroborate the relevance of collective reflection among teachers to the professional development in those two stages, and to change classroom practices toward an inclusive education.

Keywords: Inclusive education; Teacher education; Teaching practice; Group work; Collaborative reflection.

*Contacto: constanza.herrera.s@usach.cl

Recibido: 


\section{Introducción}

En la última década los estudios e iniciativas en materia de inclusión educativa han venido recalcando la importancia de asumir este desafío desde una perspectiva amplia y transformadora que reconoce la diversidad del estudiantado como un eje fundacional de la educación, no como un problema, y que aborda todas las formas de exclusión, marginación e inequidad que experimentan los estudiantes en el acceso, la participación y los resultados de aprendizaje (Florian, 2017).

En este contexto, la construcción de una pedagogía inclusiva se ha constituido en una tarea de suma importancia, que según Loreman (2017), requiere ser asumida con lentes críticos, considerando su raíz en la educación especial y el habitual uso restringido del concepto de inclusión. En dicha tarea, la formación inicial y continua del profesorado se sitúa como un elemento insoslayable, que debería permitir a las y los docentes ir más allá de una aproximación técnica de la inclusión, para ampliar su capacidad de analizar el espacio escolar desde una dimensión ética y política, así como de construcción de saberes pertinentes a la complejidad de cada contexto (Casal y Néspolo, 2019; Ocampo, 2017; Prado et al., 2018; Torres-Santomé, 2019).

En Chile, la investigación en este campo es limitada, sin embargo, los estudios existentes coinciden en que quienes estudian pedagogía, al igual que las y los profesores en ejercicio, valoran positivamente la diversidad del estudiantado, pero consideran no estar plenamente preparados para desarrollar una pedagogía inclusiva, lo que está directamente relacionado con limitaciones presentes en la formación docente inicial y continua (Araya et al., 2020; Castillo y Miranda, 2018; Valdés-Pino et al., 2021).

Dichos resultados han llevado a que se abogue por la incorporación de la inclusión en los planes de formación docente (Gelber et al., 2019; López et al., 2021). Además, se ha recalcado la importancia de fortalecer una comprensión profunda de los conceptos asociados a esta y de promover la reflexión docente acerca de las propias actitudes, prácticas pedagógicas e identidad profesional desde un enfoque inclusivo (Araya et al., 2020; Bugueño y Guzmán, 2021; Cisternas y Lobos, 2019), que permita transformar los conocimientos teóricos y conceptuales -especialmente en aspectos didácticos- en un práctica pedagógica contextualizada (Castillo y Miranda, 2018; Prado et al., 2018; San Martín et al., 2017).

Adicionalmente, las y los investigadores en el tema han precisado que la formación del profesorado debe ser abordada desde la colaboración entre profesores que, al tiempo de permitirles fortalecer su conocimiento sobre una pedagogía inclusiva, los estimule a comprender la profesión docente como un desarrollo continuo que requiere del trabajo colectivo para la movilización de cambios en las comunidades educativas (Duk et al., 2019; Durán y Gine, 2011; Valdés-Pino et al., 2021).

En atención a estos antecedentes, en este estudio de investigación-acción-formación, nos planteamos como objetivo describir el aporte de la reflexión colectiva sobre la práctica entre profesoras en formación inicial y continua para la construcción de una pedagogía inclusiva, a partir de los principios del diseño universal para el aprendizaje. A continuación, profundizaremos en los aspectos conceptuales de la propuesta, en los detalles del método empleado, así como en los resultados y conclusiones más relevantes que emanan de los datos recopilados. 


\section{Hacia la construcción de un modelo conceptual de formación docente para la inclusión}

El presente trabajo nos ha puesto el desafío de delinear un modelo conceptual que nos permita abordar la complejidad que implica la formación docente en educación inclusiva. En este contexto, al inicio del trabajo delimitamos una propuesta orientadora del diseño y desarrollo de la iniciativa que se basa en cuatro ejes: pedagogía inclusiva, vínculo entre la escuela y la universidad, trabajo colectivo entre docentes, y reflexión sobre la práctica.

Tal como se aprecia en la Figura 1, abordamos la formación docente para la inclusión, desde el enfoque de pedagogía inclusiva (Florian y Beaton, 2018; Florian y Linklater, 2010; Spratt y Florian, 2015), la que no comprendemos como un estado o cualidad docente, sino como un espacio de actuación en constante (re)construcción.

\section{Figura 1}

\section{Modelo conceptual que fundamenta la propuesta}

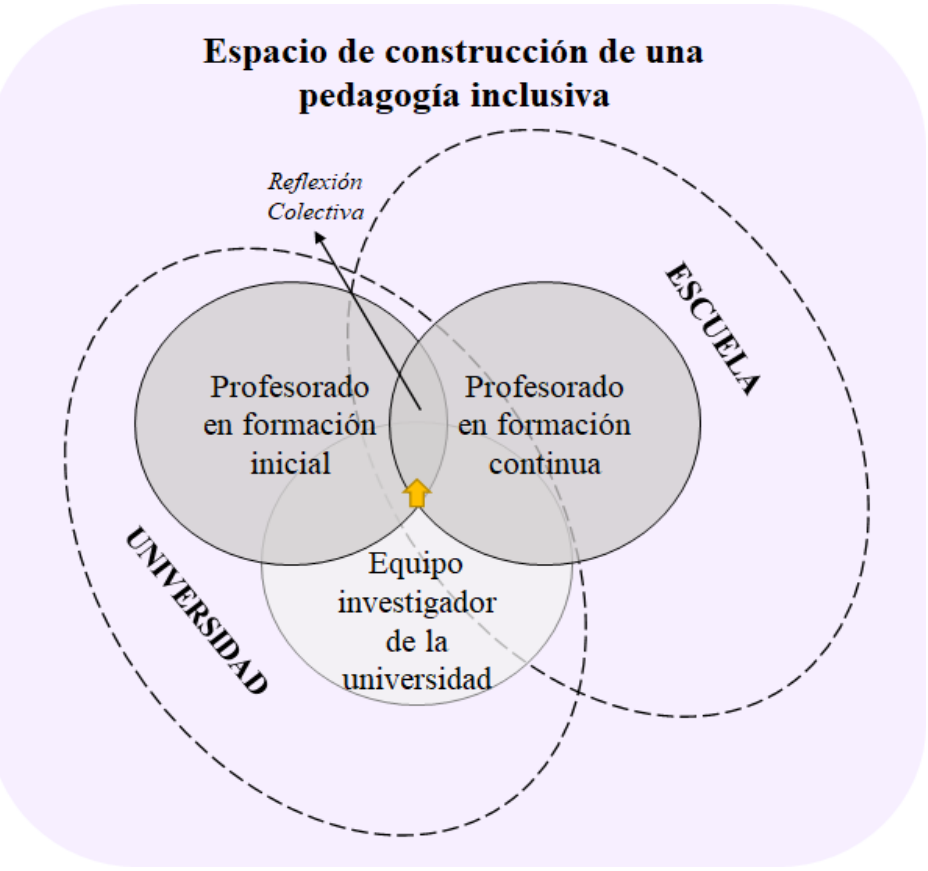

Desde una perspectiva de educación pública, consideramos que una formación docente transformadora requiere del fortalecimiento del vínculo entre la escuela y la universidad, considerando la naturaleza complementaria de sus saberes y el carácter virtuoso de dicho vínculo (Fernández et al., 2019; Tenorio et al., 2020). Por otra parte, nos ha parecido fundamental acercarnos a esta formación desde una perspectiva del trabajo colectivo entre docentes, que surge del reconocimiento de que la naturaleza dilemática y compleja de la inclusión requiere de la colaboración entre diversos actores educativos (Duk y Murillo, 2016; Echeita, 2019; Messiou, 2019).

En dicho contexto, la reflexión sobre la práctica nos resulta una dimensión fundamental que permite tejer puentes entre el posicionamiento ético en torno a la justicia social orientado a todo el estudiantado y la transformación del trabajo docente en las escuelas y las universidades (Isik-Ercan y Perkins, 2017). 
Cabe indicar, que para esta experiencia proponemos una formación integrada entre estudiantes de pedagogía y profesoras de un centro escolar. Si bien reconocemos la relevancia de las y los formadores de profesores en este proceso (García-González et al., 2018) y la riqueza que emerge de la constitución de tríadas formativas (Vanegas-Ortega y Fuentealba, 2017), optamos por fortalecer el trabajo colectivo entre profesoras en formación inicial y continua, en atención a que son quienes habitualmente comparten el trabajo en el espacio escolar. En concordancia, como equipo investigador de la universidad asumimos un rol dinamizador de la reflexión y el trabajo colectivo. Los elementos fundantes de esta propuesta, descritos en breve anteriormente, se detallarán en los siguientes apartados.

\subsection{Pedagogía inclusiva}

En este trabajo ponemos énfasis en el papel que juegan las y los profesores en los cambios a nivel de aula y de escuela. En dicho sentido, asumimos una pedagogía inclusiva que entiende el trabajo docente como un quehacer que requiere repensar y transformar continuamente los enfoques, estructuras organizativas y metodologías empleadas (Moriña, 2020), y que se orienta hacia la justicia social a partir de la provisión de apoyos accesibles universalmente, que permiten generar un rango amplio de opciones para aprender y participar, respecto de las cuales los estudiantes tienen la oportunidad de elegir (Spratt y Florian, 2015).

Desde este enfoque, las y los docentes reflexionan en torno a las barreras presentes en el currículum y en sus prácticas pedagógicas, y diseñan intencionadamente actividades de aprendizaje y evaluaciones que permiten a todos los estudiantes alcanzar aprendizajes significativos y relevantes, sobre la base de sus propias capacidades (Mohsin y Zakaria, 2017).

En Chile, la investigación en este tema ha encontrado que las y los profesores suelen tener una disposición positiva por desarrollar un enfoque inclusivo (Araya et al., 2020; Gelber et al., 2019), pero suelen emplear con escasa regularidad estrategias de enseñanza variadas, ajustadas a las características y ritmos del estudiantado, que les permitan tomar decisiones y que aseguren la participación y el aprendizaje de todos (Canales et al., 2018; Cisternas y Lobos, 2019; Gelber et al., 2019; Jiménez y Montecinos, 2018; López et al., 2021).

Finalmente, estos hallazgos se han relacionado con una serie de factores, entre los cuales destacan las limitaciones en la formación docente (Jiménez y Montecinos, 2018; San Martín et al., 2017), una cultura escolar que enfatiza la competencia, la clasificación, los contenidos y la estandarización, y condiciones materiales que dificultan el quehacer docente, como las restricciones de tiempo e infraestructura y el elevado número de estudiantes por curso (Araya et al., 2020; Cisternas y Lobos, 2019).

\subsection{Vínculo entre la escuela y la universidad}

La investigación en torno a la formación del profesorado, tanto en el ámbito de la inclusión como en otras áreas, ha mostrado la relevancia de anclar los principios teóricos con experiencias prácticas en el contexto escolar (Azorín-Abellán, 2018; Mendoza et al., 2020). En este contexto, nos ha parecido importante abordar el vínculo entre la escuela y la universidad, teniendo en cuenta que como describen Tenorio et al. (2020), las experiencias de formación que surgen de dicha relación son todavía iniciales, aun cuando en los discursos de la política pública y en las mismas instituciones se declare la relevancia de 
fortalecer dicho vínculo. Al respecto, las alianzas que se establecen entre la escuela y la universidad para favorecer el desarrollo profesional del profesorado han sido estudiadas y valoradas desde hace décadas (Ascher y Schwartz, 1989). Sin embargo, suelen ser menos frecuentes de lo deseable y más bien se expresan en intercambios aislados que se producen por necesidad o conveniencia de una de las partes (Álvarez y Osoro, 2014).

En este sentido, en la formación del profesorado ha prevalecido una racionalidad técnica que supone llevar la teoría a la práctica y que resulta poco apropiada para la comprensión del aprendizaje y la acción profesional desde la experiencia (Russell, 2012). En este contexto, resulta urgente pensar otras racionalidades a partir de las cuales el vínculo entre la escuela y la universidad permita el establecimiento de un diálogo colaborativo basado en dinámicas intergrupales donde existen relaciones equitativas de poder (Miller y Hafner, 2008) y donde se valora el aporte teórico y práctico de las y los actores de ambas instituciones, al igual que los saberes docentes que surgen de la reflexión y el trabajo colaborativo entre éstos (Guerra y Montenegro, 2017; Tenorio et al., 2020).

Sin embargo, la investigación y la formación que se desarrolla en alianza entre la escuela y la universidad está llena de dilemas, siempre resultará compleja pues en cada institución hay una realidad particular, lo que hace que no existan fórmulas únicas para llevar adelante procesos de aprendizaje que devengan en mejoras sostenidas en las instituciones educativas o las prácticas de enseñanza (Fernández et al., 2019; Vedder-Weiss et al., 2020). Lo anterior, nos ha llevado a explorar este vínculo, particularmente en relación con la construcción de una pedagogía inclusiva a partir de una formación colaborativa.

\subsection{Trabajo colectivo entre docentes}

En Chile, uno de los pilares del Sistema de Desarrollo Profesional Docente es el trabajo colectivo entre el profesorado (MINEDUC, 2018). La experiencia internacional ha apoyado con evidencias esta perspectiva (Ávalos-Bevan y Bascopé, 2017; Smith et al., $2021)$ puesto que se genera un sentido de colegialidad que conduce a querer compartir los puntos de vista, a cuidar-se profesionalmente, a tener discursos y propósitos comunes al momento de generar experiencias pedagógicas, es decir, al desarrollo una identidad profesional compartida (Vanegas-Ortega y Fuentealba, 2019).

Coherente con ello, los resultados de los estudios en América Latina han mostrado que el trabajo colectivo tiene la potencialidad de convertirse en un espacio de transformación del docente y de su práctica pedagógica (González-Weil et al., 2014; Jiménez, 2020; Jiménez et al., 2018; Vélez et al., 2019).

En nuestra investigación apostamos por el trabajo colectivo entre profesoras de la escuela y estudiantes de pedagogía, mediado por el equipo de investigación de la universidad. En ese sentido, buscamos la conformación de grupos diversos en los que las experiencias de quienes se encuentran en la formación continua se vean enriquecidas por las perspectivas de quienes se encuentran en la formación inicial, y viceversa. De esa manera, como se indicó en la Figura 1, buscamos la generación de espacios de construcción de una pedagogía inclusiva basados en la confianza, el respeto, la empatía y el diálogo mutuo.

Desde nuestro modelo conceptual, la construcción de una cultura colectiva entre el profesorado en formación inicial y continua implica atender al desarrollo profesional docente mediante un trabajo compartido, en busca de transformaciones educativas sustentadas en el análisis de la propia práctica (Hordvik et al., 2020; Ulvik et al., 2020). De esa manera, como plantean Fernández-Díaz y otros (2015), mediante espacios de 
investigación-acción-formación colaborativos es posible consolidar dinámicas de trabajo para la reflexión sobre la propia práctica docente, como andamiaje indispensable para orientar la transformación educativa.

\subsection{Reflexión sobre la práctica}

La reflexión docente es uno de los marcos conceptuales que más se han usado en los últimos años como base para el desarrollo profesional del profesorado (Isik-Ercan y Perkins, 2017; Pantić, 2021; Uştuk y De Costa, 2021; Vanegas-Ortega y Fuentealba, 2019). En concordancia, en Chile las políticas educativas se han orientado a favorecer la formación inicial y continua de un profesional reflexivo (MINEDUC, 2016, 2018). Sin embargo, como reporta la literatura, la reflexión es un concepto polisémico y con mecanismos de acción poco claros (Pantić, 2021; Vanegas-Ortega y Fuentealba, 2018).

En este modelo conceptual hemos optado por comprender la reflexión docente como la interrelación de procesos cognitivos y afectivos (Vanegas-Ortega y Fuentealba, 2018) que ocurren cuando interactúan profesores en formación continua y en formación inicial, para planificar o discutir sobre la implementación de prácticas que apunten a la educación inclusiva. Cabe indicar que éstos son procesos intencionados y conscientes (Dewey, 1933; Schön, 1983) detonados por la propuesta del equipo investigador de la universidad, quienes preparan las situaciones y preguntas que permiten la reflexión de los participantes.

En este caso, hemos privilegiado la reflexión colectiva a través de espacios de trabajo conjunto en pequeños grupos, donde se revisan las planificaciones y los acontecimientos de la clase a la luz de los principios y pautas de Diseño Universal para el Aprendizaje (Hall et al. 2012; Rose y Meyer, 2006). Posteriormente, se identifican y priorizan barreras para la presencia, la participación o el aprendizaje de todas las y los estudiantes, y finalmente, el grupo toma decisiones (Schön, 1983; Vanegas-Ortega y Fuentealba, 2018) sobre cambios a la planificación y al desarrollo de la clase, de tal manera que se pueda avanzar en la superación de las barreras identificadas.

La reflexión colectiva es complementada en grupos ampliados en los que se hacen explícitas las contribuciones del análisis de la práctica desde la perspectiva de docentes en formación. Además, hemos empleado entrevistas en profundidad para favorecer la reflexión individual, de tal manera que se puedan resignificar las experiencias docentes y los espacios de colaboración como mecanismos que contribuyen al desarrollo profesional (Isik-Ercan y Perkins, 2017; Uştuk y De Costa, 2021).

\section{Método}

\section{Enfoque metodológico}

En atención a nuestro interés en la investigación y la formación docente para una educación inclusiva, desarrollamos el presente trabajo desde un enfoque cualitativo y un diseño de investigación-acción. Tal como indica Colmenares (2012), este diseño se caracteriza por involucrar al mismo tiempo el conocimiento de la realidad social y la actuación que busca transformar dicha realidad.

En el contexto de la formación docente, la investigación-acción constituye una forma de construcción de conocimiento que surge de la participación y el diálogo horizontal entre los profesores de la universidad y la escuela, orientándose al fortalecimiento de la docencia 
y la práctica educativa (Ruiz-Bernardo et al., 2018). Particularmente, inscribimos nuestro trabajo en el marco más amplio de estudios latinoamericanos de investigación-formaciónacción que, a través de la cooperación entre investigadores, formadores y profesores, buscan la producción del saber pedagógico y la formación entre pares (Suárez y Davila, 2018).

\section{Participantes}

La investigación fue llevada a cabo con un grupo de profesoras de Santiago de Chile ${ }^{1}$. En particular, participaron 10 profesoras en formación de una universidad estatal, 9 mujeres y 1 hombre, de las cuales 8 corresponden a Pedagogía en Educación Básica y 2 a Pedagogía en Filosofía. Diseñamos la iniciativa en articulación con un curso obligatorio del plan de formación de dichas carreras, ubicado en el octavo semestre. La participación en el proyecto fue voluntaria y, por ende, las profesoras en formación inicial que no deseaban participar tuvieron la posibilidad de realizar dicho curso en otra sección.

Adicionalmente, participaron 5 profesoras de una escuela de educación básica de dependencia municipal, emplazada en un contexto empobrecido de una comuna al norte de Santiago. Las profesoras realizan clases en distintas disciplinas incluyendo Matemática, Historia, Arte, Música y Ciencias Naturales. Cabe notar que elaboramos el proyecto como una actividad de formación continua para las docentes de la escuela, y la participación en el mismo también fue de carácter voluntario.

Antes del inicio del proyecto, las participantes recibieron información detallada acerca del proceso de formación y la investigación. Asimismo, dimos a conocer los resguardos éticos del estudio y la confidencialidad de los datos. Cabe indicar que para este trabajo los nombres reales de las profesoras fueron reemplazados por pseudónimos, los que se muestran en el Cuadro 1 del apartado de resultados.

\section{Plan de formación}

El núcleo fundamental del presente proyecto corresponde a un plan de formación de profesores el cual consideró tres etapas (Figura 2). A continuación, describimos en profundidad cada una de ellas y los aspectos asociados a su implementación.

La etapa de preparación la realizamos en contexto presencial en el otoño de 2019 y consideramos el contacto con la dirección de la escuela y las jefaturas de las carreras de pedagogía, la presentación del proyecto y la firma de autorizaciones institucionales. Luego, invitamos a participar a las profesoras de la escuela y la universidad, les presentamos la iniciativa en detalle y les requerimos la firma de un consentimiento informado. Es importante indicar que cada profesora de la escuela seleccionó el grupo de estudiantes de un curso (nivel y disciplina), respecto de los cuales le parecía más relevante la reflexión desde un enfoque pedagógico inclusivo. Finalmente, en atención a las particularidades de quienes participaron y a las condicionantes de cada institución, diseñamos un plan de formación factible de ser implementado, considerando metodología, evaluación y recursos para el aprendizaje a ser empleados en las siguientes etapas.

\section{Figura 2}

\footnotetext{
${ }^{1}$ En este trabajo hemos optado por referirnos como las profesoras a quienes participaron del estudio, considerando la mayoría de mujeres y la necesidad de resguardar la confidencialidad de los datos del único profesor participante.
} 


\section{Esquema resumen del proceso de investigación-formación}

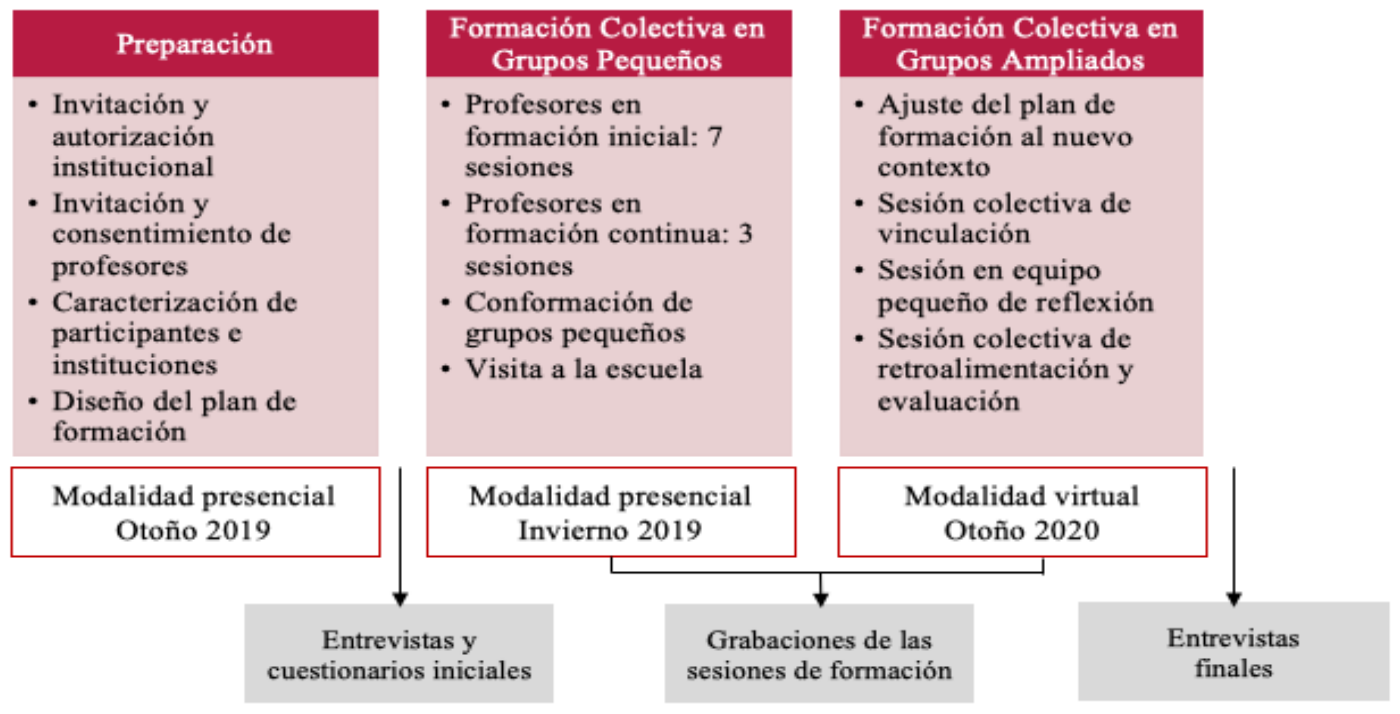

La etapa de formación colectiva en grupos pequeños la llevamos a cabo de manera presencial, en el invierno de 2019. Las actividades con las profesoras en formación inicial las desarrollamos en la universidad y contemplaron 7 sesiones de 90 minutos cada una. Por otra parte, con las profesoras en formación continua realizamos 3 sesiones de 120 minutos en la misma escuela. En ambos casos, la formación fue de carácter teórico-práctica en modalidad taller, considerando un abordaje conceptual y experiencial en torno a las temáticas de inclusión educativa, pedagogía inclusiva, reflexión docente y diseño universal para el aprendizaje. Al finalizar esta etapa conformamos al azar equipos de trabajo con dos profesoras en formación inicial y una profesora en formación continua. Cada pareja de estudiantes de pedagogía realizó una visita a la escuela en la que tuvo la oportunidad de conocer a la profesora con la que trabajaría y al grupo de estudiantes del curso seleccionado.

La etapa de formación colectiva en grupos ampliados, la habíamos planificado para la primavera de 2019. Sin embargo, suspendimos su inicio durante 6 meses, debido al estallido social en Chile a finales de 2019 y la crisis sanitaria por Covid-19 en 2020. Finalmente, logramos desarrollar esta etapa durante el otoño de 2020 en modalidad virtual. En consecuencia, previo a la realización de las sesiones de formación, adaptamos las actividades al trabajo a distancia y redujimos su extensión considerando la elevada carga académica de las profesoras de ambas instituciones.

En concreto, realizamos una primera sesión de trabajo colectivo de 60 minutos, con todas las participantes, orientada a la recuperación del vínculo, la consolidación de los aprendizajes alcanzados en las etapas anteriores y la organización del trabajo en línea. En seguida, se realizó una segunda sesión, de 90 minutos, con cada equipo por separado. Con nuestro apoyo como investigadores, reflexionamos sobre la planificación de una clase de la profesora de la escuela, considerando cuatro momentos: la revisión de la planificación original y descripción de su implementación, la identificación de barreras para la inclusión, la priorización de las barreras a ser abordadas y el rediseño de elementos de la planificación e implementación con base en los principios del Diseño Universal para el Aprendizaje. Por su parte, la tercera sesión colectiva fue desarrollada con todas las participantes y tuvo una extensión de 90 minutos. Cada equipo presentó el trabajo que había elaborado y recibió 
retroalimentación de las participantes. Asimismo, se desarrolló una actividad para una revisión general de los aprendizajes alcanzados en el proceso.

\section{Instrumentos}

En la investigación consideramos diversos instrumentos para la recolección de datos. Antes de la formación empleamos una entrevista individual, semiestructurada y en profundidad con las profesoras de la escuela, en la que abordamos la construcción de una pedagogía inclusiva considerando tres aspectos: creencias, estrategias de enseñanza y competencias percibidas. Por su parte, con las profesoras en formación inicial empleamos un cuestionario de respuesta abierta en que abordamos los 3 aspectos antes descritos y, adicionalmente, la valoración de la formación en inclusión recibida en la universidad.

Durante el desarrollo de la formación grabamos las sesiones realizadas. En las sesiones presenciales registramos mediante audio y en las sesiones a distancia a través de video. Además, las participantes sistematizaron el trabajo de reflexión en los equipos, mediante una pauta de trabajo en línea.

$\mathrm{Al}$ concluir el proceso de formación, realizamos entrevistas individuales en profundidad con las profesoras en formación inicial y continua. Elaboramos una pauta semiestructurada que, respecto a la construcción de una pedagogía inclusiva, indaga los cambios en las creencias, las estrategias de enseñanza aprendidas y la percepción de competencias luego de la formación. Asimismo, se abordaron variables institucionales, profundizando en la percepción de la formación docente y las condiciones contextuales que facilitan y dificultan la inclusión. Finalmente, incorporamos una evaluación general de la formación, la valoración del proceso de reflexión colectiva y la percepción del vínculo universidad-escuela.

\section{Análisis de datos}

En consideración a la extensión del presente trabajo, hemos optado por considerar en particular el análisis de dos dimensiones: el proceso de reflexión colectiva, que aborda lo acontecido en las sesiones de trabajo en grupos ampliados; y la construcción de una pedagogía inclusiva, que se ocupa de la evaluación de la experiencia con base en las entrevistas finales. Al respecto, utilizamos la técnica de análisis de contenido cualitativo, con ayuda del programa Atlas.ti versión 7.0, asumiendo un enfoque interpretativo que permitiera rescatar los sentidos subyacentes (Bardin, 1991; Navarro y Díaz, 2007) a los relatos docentes y los diálogos que se establecieron entre éstas.

Considerando la naturaleza de los datos, en cada dimensión realizamos el análisis con una aproximación diferente. En la dimensión reflexión colectiva utilizamos una estrategia inductiva de elaboración de códigos con base en los objetivos del estudio. Creamos 5 códigos, cada uno de los cuales se transformó en un tema: foco de la reflexión, que señala el tópico central que abordó la reflexión docente; interacciones recurrentes, que describe la naturaleza del vínculo entre las participantes; toma de decisiones, que refiere a la fuente de deliberación de las prioridades del equipo; sentidos de la profesora en formación continua, que refiere al modo como esta comprende el proceso reflexivo; y participación de las profesoras en formación inicial, que describe el rol asumido por estas en el trabajo grupal.

Por su parte, para las entrevistas empleamos una estrategia deductiva, a partir de un diccionario previamente elaborado, desde la teoría y los objetivos de la investigación, que contemplaba 10 códigos. Considerando los objetivos de este artículo abordaremos 6 de 
estos: competencias para una pedagogía inclusiva, que refiere a las habilidades docentes para abordar el desafío de la inclusión (Echeita, 2019); formación docente para la inclusión, que alude a la preparación docente en la temática (Duk et al., 2019); trabajo colaborativo, que describe las acciones emprendidas colectivamente para responder a la inclusión (Ainscow, 2019); relación universidad-escuela, que señala el vínculo de trabajo entre estas instituciones (Álvarez y Osoro, 2014); condiciones del entorno escolar, que da cuenta de las características del sistema educativo como marco de acción docente (Sisto, 2019); evaluación de la experiencia, que corresponde a la valoración sobre el proyecto; y sello de la experiencia, que puntualiza en la cualidad saliente de este tipo de formación.

Para los resultados de este artículo, consideramos el análisis de 8 horas y 26 minutos de sesiones de formación colectiva, así como 4 horas y 55 minutos de entrevistas a los participantes. Con la finalidad de garantizar la confiabilidad del estudio, realizamos procesos de triangulación entre investigadores y entre instrumentos (Piza Burgos et al., 2019). Los contenidos emanados del proceso de codificación fueron vinculados con los cuatro ejes de modelo conceptual que fundamentan la propuesta formativa, lo que nos permitió extraer inferencias de alcance descriptivo.

\section{Resultados}

\subsection{Proceso de reflexión colectiva}

En este apartado hemos decidido dar énfasis a la experiencia de reflexión colectiva que presentamos en la Figura 1, con el objetivo de visibilizar los logros, limitaciones y proyecciones de esta como medio para el desarrollo profesional docente. Lo anterior, implica poner en un segundo plano los resultados referentes a los logros alcanzados en términos del desarrollo de una pedagogía inclusiva y la utilidad del diseño universal del aprendizaje como marco de referencia para la reflexión docente.

El proceso de formación colectiva entre profesoras en formación inicial y continua fue realizado siguiendo una misma guía de trabajo en todos los casos. Sin embargo, el trabajo por equipos tuvo diferencias en su desarrollo debido a las particularidades de sus integrantes. En el Cuadro 1, se presenta una síntesis de lo ocurrido en cada equipo de profesoras a partir de los 5 temas identificados en esta dimensión.

La reflexión en el equipo 1 se focalizó en los modos a través de los cuales es posible favorecer la participación de los estudiantes, con un mayor énfasis en la didáctica disciplinar que en la inclusión del alumnado. El proceso fue percibido por la profesora en ejercicio Bárbara como un apoyo para fortalecer conocimientos e iniciativas que venía desarrollando con anterioridad. En este sentido, los aportes de las profesoras en formación inicial, Samanta y Verónica, fueron positivamente acogidos por Bárbara. Llama la atención en este equipo que las decisiones acerca del rediseño de la planificación fueron asumidas principalmente por uno de los investigadores del equipo, lo que limitó la iniciativa de las profesoras en formación. 
Cuadro 1

Características del proceso de reflexión colectiva entre profesoras por equipos

\begin{tabular}{|c|c|c|c|c|c|c|}
\hline Equipo & Participantes & Foco de la reflexión & $\begin{array}{c}\text { Interacciones } \\
\text { recurrentes }\end{array}$ & Toma de decisiones & Sentidos de la PFC & $\begin{array}{c}\text { Participación de las } \\
\text { PFI }\end{array}$ \\
\hline E1 & $\begin{array}{l}\text { Bárbara (PFC) } \\
\text { Samanta (PFI) } \\
\text { Verónica (PFI) }\end{array}$ & $\begin{array}{l}\text { Participación en la } \\
\text { clase }\end{array}$ & Apoyo & Centrada en el IN & Enriquecimiento & $\begin{array}{l}\text { Espontánea desde el } \\
\text { inicio }\end{array}$ \\
\hline $\mathrm{E} 2$ & $\begin{array}{l}\text { Esperanza (PFC) } \\
\text { Elizabet (PFI) } \\
\text { Margarita (PFI) }\end{array}$ & $\begin{array}{l}\text { Estrategias de } \\
\text { enseñanza }\end{array}$ & Apoyo & $\begin{array}{l}\text { Compartida entre la } \\
\text { PFC y los IN }\end{array}$ & Aprendizaje & $\begin{array}{l}\text { Guiada toda la } \\
\text { actividad }\end{array}$ \\
\hline E3 & $\begin{array}{l}\text { Paulina (PFC) } \\
\text { Rocío (PFI) } \\
\text { Karina (PFI) }\end{array}$ & $\begin{array}{l}\text { Actividades de la } \\
\text { clase }\end{array}$ & Información & Centrada en el IN & Evaluación & $\begin{array}{l}\text { Espontánea desde el } \\
\text { inicio }\end{array}$ \\
\hline $\mathrm{E} 4$ & $\begin{array}{l}\text { Carolina (PFC) } \\
\text { Mónica (PFI) } \\
\text { Lorena (PFI) } \\
\end{array}$ & $\begin{array}{l}\text { Actividades de la } \\
\text { clase }\end{array}$ & Información & Centrada en la PFC & Conocimiento & $\begin{array}{l}\text { Espontánea con el } \\
\text { desarrollo de la } \\
\text { actividad }\end{array}$ \\
\hline E5 & $\begin{array}{l}\text { Andrea (PFC) } \\
\text { Amanda (PFI) } \\
\text { Maite (PFC) }\end{array}$ & $\begin{array}{l}\text { Aprendizaje y } \\
\text { participación en la } \\
\text { clase }\end{array}$ & Colaboración & $\begin{array}{l}\text { Distribuida entre } \\
\text { todos }\end{array}$ & Aprendizaje & $\begin{array}{l}\text { Espontánea desde el } \\
\text { inicio }\end{array}$ \\
\hline
\end{tabular}

Nota. IN = Investigador(es); PFC = Profesora en formación continua; PFI = Profesora(s) en formación inicial. 
En el caso del equipo 2, la percepción de la profesora en ejercicio Esperanza acerca de su limitada formación pedagógica llevó a que el foco en la reflexión estuviese más en las estrategias de enseñanza que en la inclusión del estudiantado y que ésta asumiera el proceso como una instancia de aprendizaje relevante. Por su parte, la participación de las profesoras en formación inicial Elizabet y Margarita no fue espontánea, sino que incentivada por los investigadores. En este escenario, las decisiones acerca de las modificaciones a la planificación fueron compartidas entre Esperanza y los investigadores de la universidad.

Por otra parte, nos parece interesante lo ocurrido en el equipo 3, así como las similitudes y diferencias que presenta con el equipo 4. En el equipo 3 observamos que la reflexión estuvo restringida a las actividades de la clase que la profesora en ejercicio Paulina pensaba o solía realizar, sin lograr profundizar en la inclusión del estudiantado. Más aún, esta asumió el proceso como una evaluación de su desempeño, aun cuando las otras participantes mostraron una actitud respetuosa y cauta. Si bien la participación de las profesoras en formación inicial, Rocío y Karina, fue espontánea y activa, la toma de decisiones estuvo principalmente en manos de uno de los investigadores y la interacción se orientó a proveer de información a Paulina sobre los aspectos de la clase que podrían ser revisados o considerados.

Algo similar sucedió en el equipo 4, en el que la reflexión también estuvo centrada en las actividades que la profesora en ejercicio Carolina realizaba habitualmente, alejándose de la experiencia del alumnado. La profesora mostró gran interés por conocer la opinión y aportes de las profesoras en formación inicial Tamara y Lorena, lo que favoreció que estas últimas fueran adquiriendo confianza para participar de manera espontánea. Sin embargo, el diálogo del equipo fue asumido por Carolina como una fuente de información para tomar de manera individual la mayor parte de las decisiones en torno al rediseño de la clase.

Finalmente, el caso del equipo 5 fue sumamente significativo para la investigación. La profesora en ejercicio Andrea asumió el proceso de reflexión como una oportunidad de aprendizaje, a partir del reconocimiento de sus escasos conocimientos en inclusión, valorando reiteradamente los conocimientos de las profesoras en formación inicial Amanda y Maite, quienes se involucraron y participaron activamente de toda la sesión. En este equipo, hemos podido observar que hubo efectivamente colaboración, la que se orientó a fortalecer el diseño de la clase para favorecer el aprendizaje y la participación de todo el estudiantado, mediante el diálogo y la toma de decisiones distribuidas entre todos los participantes.

\subsection{Construcción de una pedagogía inclusiva}

Más allá de las interesantes diferencias que fue posible identificar en el trabajo colectivo de los equipos, el análisis de las entrevistas realizadas al finalizar el proyecto nos permitió rescatar una serie de aspectos valorados por las profesoras participantes. Con base en los temas emanados del análisis, describiremos aquellos contenidos recurrentes en los relatos y que nos han parecido interesantes para iluminar la reflexión en torno al desarrollo profesional del profesorado en un marco pedagógico inclusivo.

En cuanto al trabajo colaborativo, las profesoras en formación inicial y continua comparten que la colaboración es constitutiva de la formación y la práctica docente. Las profesoras en formación inicial señalan que la participación en este proyecto les ayudó a transformar el principio de colaboración docente, ampliamente revisado en la teoría, en una experiencia concreta a la que no suelen tener acceso en su formación práctica en las 
escuelas. Asimismo, para las profesoras en formación continua, la experiencia se articuló como una necesidad de encuentro que no suele darse en la escuela para quienes se desempeñan en áreas consideradas menos relevantes en el currículum (diferentes a matemática y lenguaje).

Al respecto, Bárbara señala que el proyecto "reafirma que como profesores tenemos que trabajar en equipo, que es súper importante el tema de las comunidades de aprendizaje, o sea compartir experiencias entre los profesores, que nos podamos observar nuestras prácticas y que nos sentemos a analizar lo que hacemos en el aula”.

En consistencia con lo anterior, resulta interesante que, en lo referente a las competencias para una pedagogía inclusiva, las profesoras en formación continua indican que la reflexión con otras docentes ha sido una oportunidad para romper el aislamiento y notar que las experiencias e inseguridades que experimentan acerca de la inclusión no son individuales sino compartidas. Sobre esto Andrea indica: "cuando mis compañeras daban sus experiencias era como ¡ah! no me pasa tan solo a mí, claro a ellas les pasa algo parecido que a mî". A lo que Paulina agrega: "sirve para darse cuenta que no es algo de una, porque igual una siente 'soy mala, no vi esto', pero al ver las clases de mis compañeras vi que pasaban un poco por lo mismo".

Nos ha llamado la atención que, respecto a la formación docente para la inclusión, las profesoras en formación inicial exponen que la participación en el proyecto fue relevante para ponerse en el lugar de aquellas docentes que se encuentran en la escuela, visualizarse en ese espacio y evaluar sus propias competencias. Adicionalmente, señalan darse cuenta de que si bien una educación inclusiva resulta un desafío complejo, no es un horizonte inalcanzable, sino que es posible en colaboración con otros.

En este sentido, Margarita indica: "Después de esta experiencia como que empecé a reflexionar bastante y cuestionarme sobre ¿cómo lograría yo quizás estas cosas? Porque criticar es super fácil, pero hacerlo, no sé, y ¡hacerlo sola!”. Por su parte, al consultar a Rocío acerca de su nivel de preparación para desarrollar una práctica pedagógica inclusiva señala: "En este momento yo diría que más o menos. O sea, me complica un poco, pero obviamente lo podré lograr con la ayuda de otros docentes. Igual el trabajo docente es un trabajo colaborativo".

En relación con la evaluación de la experiencia encontramos que, además de la oportunidad de encuentro, la valoración positiva de este proyecto surge de la disposición que han mostrado las participantes, especialmente las profesoras en formación continua, que han compartido su trabajo para la reflexión colectiva. Al respecto, la disposición implicaría tanto flexibilidad mental como apertura emocional.

Amanda, una de las profesoras en formación inicial, rescata de su experiencia en el equipo 5: "La disposición de la profesora del colegio. Siento que igual ella estuvo súper abierta. Cuando fuimos a observar su clase, fue súper amable, nos atendió bien, nos trató de explicar su contexto, como que nos hizo muy partícipes del proceso. Al igual que cuando nos tocó modificar su clase, ella en ningún momento fue negativa o criticó nuestras propuestas. Fue súper, súper y estoy agradecida de ella”.

En consecuencia, la apertura que emana de un vínculo que es eminentemente emocional, requiere del establecimiento de una relación que se construye con el tiempo. En este sentido, las participantes concuerdan en que la principal limitación que tuvo la experiencia estuvo relacionada con la escasa duración de la iniciativa producto de la contingencia 
social y sanitaria en el país. Sobre este aspecto, Karina, una de las profesoras en formación inicial, indica: "Tal vez faltó un poco más de comunicación o un poco más de cercanía. Tal vez hubiésemos podido lograr mejores cosas si nos hubiésemos extendido, si hubiésemos visto otra clase o hubiésemos ejecutado la clase y reflexionado sobre ella”.

Un último aspecto que nos gustaría abordar a propósito de los resultados de esta experiencia, corresponde a las condiciones para la construcción de una pedagogía inclusiva, las que se relacionan con los temas: condiciones del contexto escolar y relación universidad-escuela. En los relatos de las profesoras en formación se describe con claridad un marco contextual que tensiona y restringe la formación y la acción docente. Dicho marco ha sido largamente descrito en otras investigaciones chilenas (Cornejo et al. 2018; Falabella, 2021; Fernández et al., 2016; Ramírez-Casas del Valle et al. 2019; Sisto, 2019), pero creemos importante destacar tres elementos que resultan salientes en este proyecto:

- La formación de las profesoras está limitada por la falta de proyectos institucionales universidad-escuela y la insuficiente consideración de las iniciativas docentes.

- La acción de las profesoras se ve restringida por la precarización de las condiciones laborales de los docentes y el empobrecimiento de la escuela pública.

- La formación y la acción de las profesoras en inclusión se ve tensionada por políticas públicas que invisibilizan la diversidad y favorecen la discriminación.

Sin perder de vista este marco y una serie de otras condicionantes que hemos observado durante el proceso, el análisis del sello de la experiencia nos muestra que la reflexión colectiva entre profesoras con motivo de pensar en una pedagogía inclusiva (Figura 3) teje puentes entre la teoría que suele dominar la formación -inicial y continua- de las docentes, y la práctica que cruza las experiencias de los distintos actores de la comunidad educativa.

\section{Figura 3} Elementos claves que dinamizan el espacio de construcción de una pedagogía
inclusiva

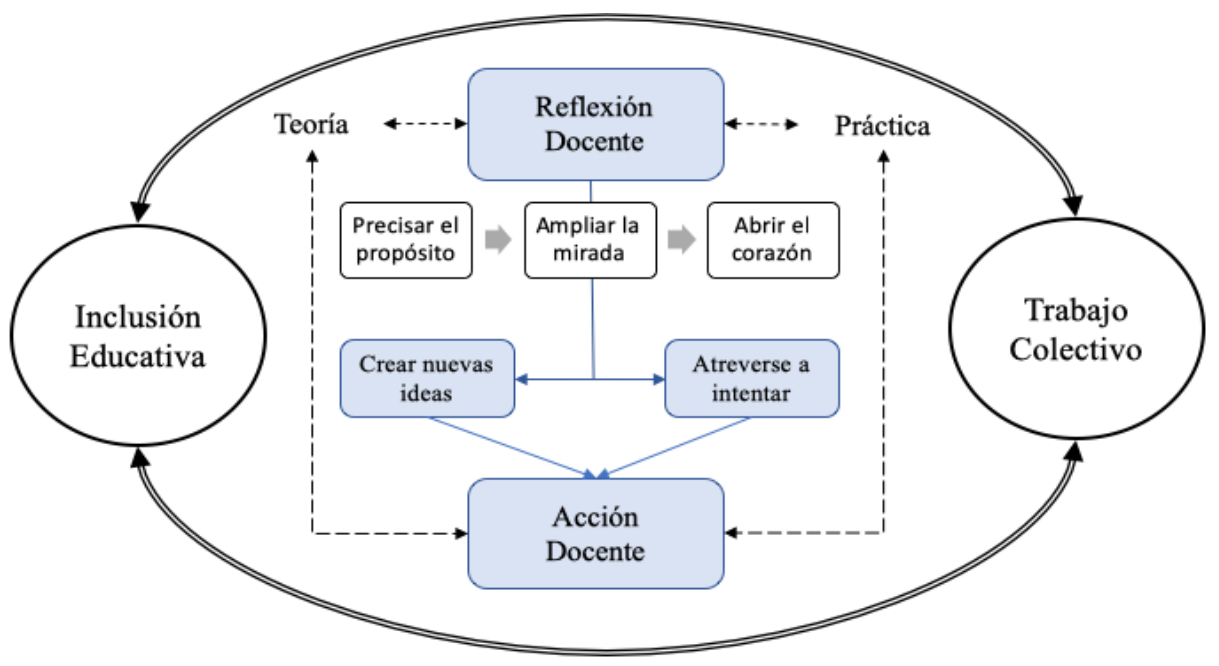

A partir de esta reflexión es que las profesoras han podido precisar el propósito, desde una perspectiva inclusiva comprehensiva que se distingue de otros enfoques del cambio en la escuela, que al mismo tiempo las ha llevado a ampliar la mirada respecto a la diversidad 
del estudiantado y al rol docente en la inclusión, y las ha llevado a abrir el corazón estando dispuestas a reconocerse como profesoras en un proceso de aprendizaje sin fin. De dicha reflexión sobre la práctica surgen nuevas ideas y también la determinación de atreverse a intentar transformar el aula, movilizando la acción docente y abriendo nuevos espacios de reflexión que permiten la constante re-construcción de una pedagogía inclusiva.

Al respecto, podemos rescatar el relato de Samanta, una de las profesoras en formación inicial, cuando describe la relevancia de esta experiencia en comparación a otras instancias de formación en inclusión en la universidad: "Con estas profesoras en particular, aprendí cómo llevarlo a la práctica, porque a pesar de todos mis conocimientos en inclusión, con ellas tuve más experiencias, de saber cómo ellas lo habían interiorizado y de cómo ellas lo habían hecho posible en su sala de clases, entonces fue súper significativo”.

Finalmente, resulta interesante lo planteado por Carolina cuando evalúa el proyecto y lo compara con formaciones anteriores en inclusión: "A mí me gustó mucho el trabajo con las chiquillas, el análisis de la planificación, escuchar las opiniones de las colegas [profesoras en formación inicial] y de mis colegas [profesoras en formación continua]. Estuvimos ahí ayudándonos a realizar este trabajo, fue muy bueno, fue muy útil. Prefiero esta situación que no es tan lejana, es más cercana”.

\section{Discusión y conclusiones}

El desarrollo de este proyecto de investigación-acción-formación ha resultado fructífero no sólo en términos de explorar la dinamización de un espacio de construcción colectiva de la pedagogía inclusiva o por los aportes para sus participantes, sino también porque nos ha permitido identificar una serie de debilidades y también algunas fortalezas de la propuesta, que contribuyen a delinear las condiciones requeridas para el desarrollo de procesos de formación docente efectivamente transformadores a nivel personal y escolar. En lo que sigue enfatizamos aquellos resultados que nos han parecido especialmente provocadores en términos de las interrogantes que nos sugieren y los desafíos futuros que nos plantean.

Los relatos de las profesoras en formación inicial y continua nos han permitido corroborar la relevancia de la reflexión colectiva entre docentes para el desarrollo profesional y la transformación de las prácticas de aula hacia la inclusión educativa (Isik-Ercan y Perkins, 2017; Pantić, 2021; Uştuk y De Costa, 2020; Vanegas-Ortega y Fuentealba, 2019). Particularmente, hemos observado la relación virtuosa que surge entre el trabajo colectivo y la inclusión. Al respecto, la inclusión le otorga a la colaboración entre profesoras un horizonte, un sentido ético y político de justicia educativa. En coherencia con otros trabajos (Azorín-Abellán, 2018; Florian y Beaton, 2018; Valdés-Pino et al., 2021), dicho colectivo docente se transforma a medida que la reflexión sobre la práctica pueda detectar focos específicos de acción que permitan vehiculizar la construcción de una pedagogía inclusiva.

En tal sentido, consideramos que, como señalan Álvarez y Osoro (2014) cuando evalúan la experiencia de investigación-acción que han desarrollado, no sería exagerado afirmar que el presente trabajo constituye una experiencia de desarrollo profesional tanto en la formación inicial como continua, con miras de abordar el reto de la educación inclusiva, en la cual se ubica la reflexión colectiva entre docentes en el centro de la propuesta. 
Sin embargo, como hemos descrito ampliamente a la luz de lo ocurrido en los equipos, debemos ser cautos al valorar los aportes de la experiencia, pues más allá de sus contribuciones generales, los logros específicos al interior de cada grupo estuvieron marcados por una serie de factores entre los que destacan la motivación y disposición de quienes participaron, la autopercepción de las profesoras acerca sus competencias pedagógicas, las dinámicas que surgen a partir de diferentes estilos de dinamización grupal, entre otros.

Uno de los aspectos analizados en los que existe acuerdo entre las profesoras es la relevancia de la colaboración docente, tal como anteriormente lo han descrito un sinnúmero de trabajos en educación inclusiva (Ainscow, 2019; Duk et al., 2019; Echeita, 2019).

Nos parece interesante constatar que, tanto en las profesoras en formación inicial como aquellas en ejercicio, el encuentro y diálogo con otras profesoras supone compartir experiencias que otorgan seguridad a la práctica docente. Para las profesoras que se inician, se trata de una oportunidad que abre la posibilidad de abordar los múltiples desafíos del trabajo docente y educación inclusiva junto a otras. Para aquellas que se encuentran en ejercicio, constituye una instancia de mutuo reconocimiento, que permite compartir vivencias, aprender mutuamente y disipar preocupaciones acerca de las dificultades que surgen al intentar orientar la práctica teniendo en cuenta la diversidad del estudiantado.

Tal como expone Florian (2017), desarrollar el trabajo docente desde el imperativo de construir una escuela para todos, tiene importantes repercusiones en la formación del profesorado. A partir de los resultados de este trabajo podemos identificar al menos dos: la necesidad de fortalecer las capacidades del profesorado para participar de procesos de reflexión colaborativa y de generar las condiciones en las instituciones educativas que propicien y hagan sostenibles tales procesos.

En concordancia con lo anterior, otro aspecto que nos parece relevante de los resultados es que las diferencias en la disposición mental y emocional de las profesoras de la escuela para involucrarse en la reflexión sobre su práctica, influyó en la participación de las profesoras en formación inicial. Lo que ratifica la atención que se debe prestar a los componentes afectivos de los procesos reflexivos (Dewey, 1933; Schön, 1983; VanegasOrtega y Fuentealba, 2018). En este sentido, en los equipos donde las profesoras en formación continua mostraron mayor disposición a aprender o enriquecer sus diseños y acciones, se logró relaciones de ayuda o colaboración con las profesoras en formación inicial, a partir del reconocimiento mutuo de los saberes de cada participante.

Sin embargo, tal como hemos indicado con anterioridad, probablemente una de las limitaciones más relevantes de esta experiencia estuvo en el tiempo reducido de la formación. En consecuencia, nos cuestionamos si en iniciativas posteriores de mayor extensión sería posible favorecer una mayor disposición y un vínculo de confianza que promueva aprendizajes y transformaciones sostenibles de la práctica docente desde un enfoque inclusivo. Además, nos parece que valdría la pena profundizar en las trayectorias de desarrollo profesional que llevan a algunas profesoras a mostrar mayor disposición, rescatando las particularidades de los diversos contextos formativos y laborales que la favorecen.

Por otro lado, el análisis minucioso de la experiencia nos ha desafiado en nuestro propio rol como investigadores y formadores de profesores (García-González et al., 2018). Si bien 
reconocemos los aportes de la propuesta de formación elaboradora y nuestra contribución en la dinamización del trabajo de los grupos, hemos logrado identificar que las diferencias en lo acontecido en los equipos pequeños, está condicionado también por nuestros propios posicionamientos y competencias sobre la educación inclusiva y sobre la reflexión, así como por la experiencia que fuimos acumulando como equipo a lo largo del trabajo con las profesoras.

Sobre este punto nos parece fundamental avanzar en dos direcciones. Primero, creemos importante incorporar en trabajos venideros un registro detallado de las experiencias, creencias y acciones de los investigadores como sujetos individuales y del equipo de investigación como actor colectivo, con la finalidad de enriquecer la comprensión de este espacio de construcción pedagógica desde una reflexión y análisis que se da en primera persona y al que los estudiosos de los métodos biográfico-narrativos en la investigaciónacción-formación le han otorgado tanta importancia (Delory-Momberger, 2020; Suárez y Dávila, 2018).

Segundo, consideramos urgente asumir una perspectiva crítica respecto a nuestro accionar como investigadores y formadores, orientando nuestro trabajo a la construcción de capacidades al interior de las escuelas, que permita a los profesores que allí se desempeñan movilizar procesos de investigación y reflexión, para su autoformación y el apoyo a los profesores en formación inicial. En esta construcción, sin duda una de las tareas más relevantes será sortear las dificultades impuestas por las condiciones contextuales que constriñen la acción del profesorado y que hemos descrito en los resultados.

Finalmente, quisiéramos notar que al analizar los datos que hemos recopilado, el vínculo entre la escuela y la universidad tiende a diluirse, incluso al consultar a las profesoras directamente por este. Sin embargo, la ausencia de referencias a esta alianza en términos institucionales contrasta con la valoración que las participantes le otorgan al establecimiento de este vínculo en la dimensión de la experiencia que encarnan sus actores.

En consecuencia, nos parece relevante proyectar la investigación y formación del profesorado, por una parte, en el sentido de profundizar en la comprensión de la agencia docente que se ha venido trabajando en los últimos años en materia de inclusión (Pantić, et al. 2021; Pantić y Florian, 2015), por otro, con miras a revisar las políticas públicas e institucionales de formación docente, a fin de estudiar el aporte que el establecimiento de vínculos institucionales entre la escuela y universidad (Russell, 2012) podría tener en el fortalecimiento y la ampliación de experiencias como la que hemos descrito en este trabajo.

\section{Agradecimientos}

El presente trabajo se enmarca en el Proyecto de Innovación Docente $\mathrm{N}^{\circ} \mathrm{O} 11-2018$ titulado "Diseño de secuencias didácticas inclusivas en colaboración con la escuela. Una experiencia de aprendizaje de servicio en Pedagogía en Educación General Básica, financiado por la Vicerrectoría Académica de la Universidad de Santiago.

\section{Referencias}

Ainscow, M. (2019). 25 años después de la Declaración de Salamanca de la UNESCO. Crear sistemas educativos inclusivos y equitativos. UNESCO. 
Álvarez, C. y Osoro, J. M. (2014). Colaboración universidad-escuela para el cambio escolar. Una investigación-acción en proceso. Innovación Educativa, 24, 215-227. https://doi.org/10.15304/ie.24.1483

Araya, A., Álvarez, Y., Garín, L., Pallero, F., Puchi, N. y Véliz, B. (2020). Actitudes, concepciones y prácticas inclusivas de docentes y educadores diferenciales de escuelas rurales. Revista INTEREDU. Investigación Sociedad y Educación, 2(3), 61-81. https://doi.org/10.32735/S2735-65232020369

Ascher, C. y Schwartz, W. (1989). School-college alliances: Benefits for low-income minorities. ERIC/CUE Digest, 53, 33-64.

Ávalos-Bevan, B. y Bascopé, M. (2017). Teacher informal collaboration for professional improvement: Beliefs, contexts, and experience. Education Research International, 2017, 1-13. https://doi.org/10.1155/2017/1357180

Azorín-Abellán, C. M. (2018). The journey towards inclusion: Exploring the response of teachers to the challenge of diversity in schools. Revista Colombiana de Educación, 75, 39-58.

https://doi.org/10.17227/rce.num75-8100

Bardin, L. (1991). Análisis de contenido. Ediciones Akal.

Bugueño, F. y Guzmán, D. (2021). Procesos inclusivos de la educación parvularia desde la mirada de agentes educativas. Revista Actualidades Investigativas en Educación, 21(1), 1-28. https://doi.org/10.15517/aie.v21i1.42517

Canales, P., Aravena, O., Carcamo-Oyarzun, J., Lorca, J. y Martínez-Salazar, C. (2018). Prácticas pedagógicas que favorecen u obstaculizan la inclusión educativa en el aula de educación física desde la perspectiva del alumnado y profesorado. Retos, 34, 212-217. https://doi.org/10.47197/retos.v0i34.59620

Casal, V. y Néspolo, M. J. (2019). Formación de educadores: la narración como eje del saber sensible para la inclusión. En V. Casal y M. J. Néspolo (Comps). Formación de educadores para la inclusión educativa. Posiciones, miradas, recorridos y experiencias (pp. 55-62). Lugar.

Castillo, P. y Miranda, C. (2018). Actitud hacia la inclusión de los estudiantes de pedagogía de una universidad estatal chilena. Revista Latinoamericana de Educación Inclusiva, 12(2), 133-148. https://doi.org/10.4067/S0718-73782018000200133

Cisternas, T. y Lobos, A. (2019). Profesores nóveles de enseñanza básica: Dilemas, estrategias y obstáculos para abordar los desafíos de una educación inclusiva. Revista Latinoamericana de Educación Inclusiva, 13(1), 37-53. https://doi.org/10.4067/So718-73782019000100037

Colmenares, A. (2012). Investigación-acción participativa: Una metodología integradora del conocimiento y la acción. Voces y Silencios: Revista Latinoamericana de Educación, 3(1), 102115. https://doi.org/10.18175/vys3.1.2012.07

Cornejo, R. (2018). Políticas y reformas escolares: el experimento educativo chileno y su evolución. En C. Ruiz-Schneider, L. Reyes Jedliki y F. Herrera (Eds.), Privatización de lo público en el sistema escolar (pp. 237-263). LOM.

Delory-Momberger, C. (2020). Aprendizaje biográfico y formación. Márgenes, Revista de Educación de la Universidad de Málaga, 1(3), 6-15. https://doi.org/10.24310/mgnmar.v1i3.9770

Dewey, J. (1933). How we think. Heath.

Duk, C., Cisternas, T. y Ramos, L. (2019). Formación docente desde un enfoque inclusivo. A 25 años de la Declaración de Salamanca, nuevos y viejos desafíos. Revista Latinoamericana de Educación Inclusiva, 13(2), 91-109. https://doi.org/10.4067/So718-73782019000200091 
Duk, C. y Murillo, F. J. (2016). La inclusión como dilema. Revista Latinoamericana de Educación Inclusiva, 1O(1), 11-14. https://doi.org/10.4067/S0718-73782016000100001

Durán, D. y Giné, C. (2011) La formación del profesorado para la educación inclusiva: Un proceso de desarrollo profesional y de mejora de los centros para atender la diversidad. Revista Latinoamericana de Educación inclusiva, 5(2), 153-170.

Echeita, G. (2019). Educación inclusiva. El sueño de una noche de verano. Octaedro.

Falabella, A. (2021). Going left, or right? A study of the policy rationale of the Chilean center-left coalition concertación in education. Education Policy Analysis Archives, 29(5), 61-84. https://doi.org/10.14507/epaa.29.5266

Fernández, R., Albornoz, N., Cornejo, R. y Etcheberriagaray, G. (2016). Los discursos sobre autonomía del trabajo docente en el nuevo marco regulatorio educativo chileno. Currículo sem Fronteiras, 16(2), 283-302.

Fernández, R., Guerrero, G., Abricot, N. y Espinoza. L. (2019). Transferencia de la investigación asociativa en la formación inicial de profesores. En G. Guerrero, R. Fernández y G. Watson (Eds.) Investigando juntos. Experiencias asociativas entre escuelas y la Universidad de Santiago de Chile (pp. 137-143). USACH.

Fernández-Díaz, E., Gutiérrez-Esteban, P. y Fernández, L. (2015). Una investigación colaborativa interuniversitaria para repensar un modelo docente comprometido con el cambio educativo. Revista Interuniversitaria de Formación del Profesorado, 29(1), 143-155.

Florian, L. (2017). The heart of inclusive education is collaboration. Pedagogika, 126(2), 248-253. https://doi.org/10.15823/p.2017.32

Florian, L. y Beaton, M. (2018). Inclusive pedagogy in action: Getting it right for every child. International Journal of Inclusive Education, 22(8), 870-884. https://doi.org/10.1080/13603116.2017.1412513

Florian, L. y Linklater, H. (2010). Preparing teachers for inclusive education: Using inclusive pedagogy to enhance teaching and learning for all. Cambridge Journal of Education, 4O(4), 369-386. https://doi.org/10.1080/0305764X.2010.526588

García-González, C., Herrera-Seda, C. y Vanegas-Ortega, C. (2018). Competencias docentes para una pedagogía inclusiva. Consideraciones a partir de la experiencia con formadores de profesores chilenos. Revista Latinoamericana de Educación Inclusiva, 12(2), 149-167. https://doi.org/10.4067/SO718-73782018000200149

Gelber, D., Treviño, E., Escribano, R., González, A. y Ortega, L. (2019). Del dicho al hecho: Creencias y prácticas inclusivas en establecimientos y aulas escolares en Santiago. Perspectiva Educacional, 58(3), 73-101. https://doi.org/10.4151/07189729-Vol.58-Iss.3-Art.967

González-Weil, C., Waring, M. G., Ahumada, G., Cisternas, A., Pérez, J. L. y Valenzuela, J. S. (2014). Contribución del trabajo colaborativo en la reflexión docente y en la transformación de las prácticas pedagógicas de profesores de ciencia escolares y universitarios. Pensamiento Educativo, 51(2), 75-85. https://doi.org/10.7764/PEL.51.2.2014.6

Guerra, P. y Montenegro, H. (2017). Conocimiento pedagógico: Explorando nuevas aproximaciones. Educação e Pesquisa, 43, 663-680. https://doi.org/10.1590/S1517-9702201702156031

Hall, T., Meyer, A. y Rose, D. (2012). Universal design for learning in the classroom: Practical applications. Guilford Press. 
Hordvik, M., MacPhail, A. y Ronglan, L. T. (2020). Developing a pedagogy of teacher education using self-study: A rhizomatic examination of negotiating learning and practice. Teaching and Teacher Education, 88, 102969. https://doi.org/10.1016/j.tate.2019.102969

Isik-Ercan, Z. y Perkins, K. (2017). Reflection for meaning and action as an engine for professional development across multiple early childhood teacher education contexts. Journal of Early Childhood Teacher Education, 38(4), 342-354. https://doi.org/10.1080/10901027.2017.1394935

Jiménez, M. (2020) La inserción profesional docente en Colombia: Retos para la formación inicial y continua de maestros. Formação Docente. Revista Brasileira de Pesquisa sobre Formação de Professores, 12(23), 53-66. https://doi.org/10.31639/rbpfp.v\%vi\%i.287

Jiménez, M., Cividini, M., Mejía, L., Morales, A. y Pérez, M. A. (2018). Y llega uno y se estrella con un montón de cosas: La inserción profesional de profesores de ciencias naturales. Universidad de Antioquia.

Jiménez, F. y Montecinos, C. (2018). Diversidad, modelos de gestión y formación inicial docente: Desafíos formativos desde una perspectiva de justicia social. Revista Brasileira de Educacao, 23, 1-2 1. https://doi.org/10.1590/s 1413-24782018230005

López, T., Castillo, C., Taruman, J. y Urzúa, A. (2021). Prácticas inclusivas centradas en el aprendizaje: Un estudio de casos múltiples en educación infantil. Revista Educación, 45(1), 104-120. https://doi.org/10.15517/revedu.v45i1.40536

Loreman, T. (2017). Pedagogy for inclusive education. En G. W. Noblit (Ed.) Oxford research encyclopedia of education. Oxford University Press. https://doi.org/10.1093/acrefore/9780190264093.013.148

Mendoza, M., Drouilly, N. y Covarrubias, C. G. (2020). Dimensiones formativas del prácticum de pedagogía en educación general básica. Estudios Pedagógicos, 46(2), 139-157. https://doi.org/10.4067/So7 18-07052020000200139

Messiou, K. (2019). The missing voices: Students as a catalyst for promoting inclusive education. International Journal of Inclusive Education, 23(7-8), 768-781. https://doi.org/10.1080/13603116.2019.1623326

Miller, P. M. y Hafner, M. M. (2008). Moving toward dialogical collaboration: A critical examination of a university-school-community Partnership. Educational Administration Quarterly, 44(1), 66-110. https://doi.org/10.1177/0013161X07309469

MINEDUC. (2016). Ley $N^{o} 20.903$ Crea el sistema de desarrollo profesional docente y modifica otras normas. Biblioteca del Congreso Nacional de Chile.

MINEDUC. (2018). Modelo de formación para el desarrollo profesional docente y directivo. Gobierno de Chile.

Mohsin, M. y Zakaria, A. R. (2017). Inclusive pedagogy literacy among academic community at institute of teacher education, Kuala Lumpur. Open Journal of Social Sciences, 5, 114-122. https://doi.org/10.4236/jss.2017.52011

Moriña, A. (2020). Approaches to inclusive pedagogy: A systematic literature review. Pedagogika, 140(4), 134-154. https://doi.org/10.15823/p.2020.140.8

Navarro, P. y Díaz, C. (2007). Análisis de contenido. En J. M. Delgado y J. Gutiérrez (Eds.), Métodos y técnicas cualitativas de investigación en ciencias sociales (pp. 177-221). Síntesis.

Ocampo, A. (2017). Educación inclusiva crítica y exploración del espacio de la exclusión: Relevando el potencial crítico de la descolonización epistémica y los estudios de performance como ejes 
analíticos. En A. Pérez (Eds.), Perspectivas críticas acerca de la discapacidad. Aportes de la filosofía y de las ciencias sociales (pp. 57-79). Universidad Nacional de Avellaneda.

Pantić, N. (2021). Teachers' reflection on their agency for change (TRAC): A tool for teacher development and professional inquiry. Teacher Development, 25(2), 136-154. https://doi.org/10.1080/13664530.2020.1868561

Pantić, N. y Florian, L. (2015). Developing teachers as agents of inclusion and social justice. Education Inquiry, 6(3), 333-351. https://doi.org/10.3402/edui.v6.27311

Pantic, N., Galey, S., Florian, L., Joksimovic, S., Viry, G., Gasevic, D., Knutes, H. y Kyritsi, K. (2021). Making sense of teacher agency for change with social and epistemic network analysis. Journal Educational Change. https://doi.org/10.1007/s 10833-02 1-09413-7

Piza Burgos, N. D., Amaiquema Márquez, F. A. y Beltrán Baquerizo, G. E. (2019). Métodos y técnicas en la investigación cualitativa. Algunas precisiones necesarias. Conrado, 15(70), 455-459.

Prado, D., Díaz, N. y Vásquez, C. (2018). Importancia de la formación inicial docente como facilitador de prácticas inclusivas en Educación. Revista de Educación Inclusiva, 2(2), 141-174. https://doi.org/10.17227/01224328.1382

Ramírez-Casas del Valle, L., Baleriola, E., Sisto, V. y Aguilera, F. (2019). Las políticas de evaluación y sus efectos en el quehacer docente: más allá de la organización y la gestión. Paulo Freire. Revista de Pedagogía Crítica, 17(22), 29-48. https://doi.org/10.25074/07 195532.22.1447

Rose, D. y Meyer, A. (2006). A practical reader in universal design for learning. Harvard Education Press. https://doi.org/10.14507/er.v0.953

Ruiz-Bernardo, P., Sánchez-Tarazaga, L. y Mateu-Pérez, R. (2018). La innovación pedagógica de la mano de la investigación-acción para mejorar la calidad de las prácticas externas de los grados de maestro/a en educación primaria -educación infantil. Revista Electrónica Interuniversitaria de Formación del Profesorado, 21(1), 33-49. http://dx.doi.org/10.6018/reifop.21.1.277681

Russell, T. (2012). Paradigmatic changes in teacher education: The perils, pitfalls, and unrealized promise of the reflective practitioner. Encounters on Education, 13, 71-91. https://doi.org/10.24908/eoe-ese-rse.v13io.4426

San Martín, C., Villalobos, C., Muñoz, C. y Wyman, I. (2017). Formación inicial docente para la educación inclusiva. Análisis de tres programas chilenos de pedagogía en educación básica que incorporan la perspectiva de la educación inclusiva. Calidad en la Educación, 46, 20-52. https://doi.org/10.4067/SO718-45652017000100020

Schön, D. A. (1983). The reflective practitioner: How professionals think in action. Basic books.

Sisto, V. (2019). Inclusión “a la chilena”: La inclusión escolar en un contexto de políticas neoliberales avanzadas. Archivos Analíticos de Políticas Educativas, 27(23), 1-21. https://doi.org/10.14507/epaa.27.3044

Smith, A., McBride, C. y Rogers, C. (2021). Exploring the edges of collegiality: a cross-case analysis toward humanizing teachers connected learning. Pedagogies: An International Journal, 16(2), 202-2 19. https://doi.org/10.1080/1554480x.2021.1919117

Spratt, J. y Florian, L. (2015). Inclusive pedagogy: From learning to action. Supporting each individual in the context of 'everybody'. Teaching and Teacher Education, 49, 89-96. https://doi.org/10.1016/j.tate.2015.03.006 
Suárez, D. H. y Dávila, P. V. (2018). Documentar la experiencia biográfica y pedagógica. La investigación narrativa y (auto) biográfica en educación en Argentina. Revista Brasileira de Pesquisa (Auto)biográfica, 3(8), 350-373.

Tenorio, S., Jardi, A., Puigdellívol, I. y Ibañez, N. (2020). Intersección escuela-universidad: Un espacio híbrido de colaboración para fortalecer la formación inicial y el desarrollo profesional docente. Perspectiva Educacional, 59(2), 88-110. https://doi.org/10.4151/07189729-Vol.59-Iss.2-Art.1071

Torres-Santomé, J. (2019). Formación del profesorado y educación como proyecto político e inclusivo. Educação E̊ Realidade, 44(3). https://doi.org/10.1590/2175-623684911

Ulvik, M., Eide, H., Eide, L., Helleve, I., Jensen, V. S., Ludvigsen, K. y Torjussen, L. (2020). Teacher educators reflecting on case-based teaching. A collective self-study. Professional Development in Education, 46(1), 21-35. https://doi.org/10.1080/19415257.2020.1712615

Uştuk, O. y De Costa, P. I. (2020). Reflection as meta-action: Lesson study and EFL teacher professional development. TESOL Journal, 12, 1-12. https://doi.org/10.1002/tesj.531

Valdés-Pino, M. A., Calvo-Álvarez, M. I. y Martínez-Abad, F. (2021). Formación inclusiva del profesorado de primaria en tres regiones chilenas. ALTERIDAD. Revista de Educación, 16(1), 117-129. https://doi.org/10.17163/alt.v16n1.2021.09

Vanegas-Ortega, C. y Fuentealba, R. (2017). Triadic thoughtful table. Space to generate reflexive thoughts during initial training teaching practices. International Journal of Advancement in Engineering Technology, Management and Applied Science, 4(12), 19-34. https://doi.org/10.1080/13573322.201 1.589648

Vanegas-Ortega, C. y Fuentealba, A. (2018). Reflexión docente: Perspectivas teóricas, críticas y modelos para el desarrollo profesional de profesores. Appris.

Vanegas-Ortega, C. y Fuentealba, A. (2019). Identidad profesional docente, reflexión y práctica pedagógica: Consideraciones claves para la formación de profesores. Perspectiva Educacional, 58(1), 115-138. https://doi.org/10.4151/07189729-vol.58-iss.1-art.780

Vedder-Weiss, D., Lefstein, A., Segal, A. y Pollak, I. (2020). Dilemmas of leadership and capacity building in a research-practice partnership. Teachers College Record, 122(9), 1-30.

Vélez, A., González-Weil, C. y Bravo, P. (2019). Ciclos reflexivos y comunidad de aprendizaje: Hacia el cambio en la práctica pedagógica en docentes de ciencias naturales. Revista de Innovación en Enseñanza de las Ciencias, 2(1), 74-98. https://doi.org/10.5027/reinnec.V2.I 1.34

\section{Breve CV de los/as autores/as}

\section{Constanza Herrera-Seda}

Académica Departamento de Educación, Facultad de Humanidades, Universidad de Santiago de Chile. Psicóloga, Magister en Investigación Social y Desarrollo, y Doctora en Psicología de la Universidad de Concepción (Chile). Su trabajo se orienta a la formación del profesorado y profesionales en el área de la educación de pregrado y postgrado, así como al desarrollo de proyectos de investigación, innovación y vinculación con el medio en el área de educación. Su principal área de investigación se encuentra en la formación del profesorado para abordar el desafío de la inclusión educativa, considerando profesores en formación inicial, docentes en ejercicio en las escuelas y formadores del profesorado. Email: constanza.herrera.s@usach.cl 
ORCID ID: https://orcid.org/0000-0001-5778-6495

\section{Carlos Vanegas Ortega}

Académico Departamento de Matemática y Ciencia de la Computación, Facultad de Ciencia, Universidad de Santiago de Chile, Doctor en Ciencias de la Educación y Magíster en Ciencias de la Educación de la Pontificia Universidad Católica de Chile; Magíster en Educación en la línea de Ciencias Experimentales y Matemáticas de la Universidad de Antioquia (Colombia); Profesor de Matemáticas y Física de la Universidad de Antioquia. Se ha desempeñado en la educación básica y media, y en los últimos años, se ha dedicado a la formación de profesores a nivel de pregrado y posgrado. Su principal área de investigación es el desarrollo profesional docente, particularmente interesado en el pensamiento reflexivo del profesor, la identidad profesional docente, las prácticas pedagógicas, la inserción e inducción del profesorado principiante. Email: carlos.vanegas.o@usach.cl

ORCID ID: https://orcid.org/0000-0002-5364-0664

\section{Esteban Vicencio Callejas}

Egresado Pedagogía en Historia y Ciencias Sociales, Departamento de Historia, Facultad de Humanidades, Universidad de Santiago de Chile. Miembro del Centro de Estudios Migratorios de la misma casa de estudios, lo que le ha permitido profundizar y especializarse en sus campos de acción. Además, se ha desempeñado como asistente en proyectos de investigación en líneas de inclusión, formación ciudadana y migraciones. Asimismo, ha cumplido labores en programas de especialización en inclusión e investigación- acción para el desarrollo profesional del profesorado en ejercicio. Sus líneas de interés para el desarrollo de procesos de investigación, innovación y formación son la inclusión educativa, formación ciudadana y la formación continua del profesorado. Email: esteban.vicencio@usach.cl

ORCID ID: https://orcid.org/0000-0003-1369-1755

\section{Katalina Maldonado Amaro}

Estudiante de último semestre de Pedagogía en Historia y Ciencias Sociales, Departamento de Historia, Facultad de Humanidades, Universidad de Santiago de Chile. Becaria AUGM pasantía estudiantil 2017 a la Facultad de Filosofía y Letras de la Universidad de Buenos Aires, Argentina. A lo largo de su formación ha colaborado en distintos proyectos de investigación y de vinculación con el medio en educación, así como en variadas ayudantías de cátedras en el área que le permiten reafirmar su compromiso con la justicia educativa. Su principal área de interés en investigación es inclusión educativa y saberes emocionales docentes, en particular, en los últimos años ha enfocado sus procesos formativos en temáticas de trabajo y profesión docente, y la formación inicial. Email: katalina.maldonado@usach.cl

ORCID ID: https://orcid.org/0000-0003-1369-1755 